\title{
El injerto como alternativa para mejorar el rendimiento en la producción de frijol ejotero (Phaseoulus vulgaris L.)
}

\author{
Grafting vegetables as alternative for increase \\ yields on green beans (Phaseoulus vulgaris $L$.)
}

\author{
José Bernal-Alzate ${ }^{1}$, Onécimo Grimaldo-Juarez*1, Daniel Gonzalez-Mendoza ${ }^{1}$, \\ Lourdes Cervantes-Díaz ${ }^{1}$,Edgar Omar Rueda-Puente ${ }^{2}$, Carlos Ceceña-Durán ${ }^{1}$
}

\section{RESUMEN}

\begin{abstract}
El ejote es una hortaliza que forma parte de la dieta básica en Latinoamérica, sin embargo, su producción se ve frenada por diversos factores y el sector productivo demanda nuevas alternativas para el manejo de enfermedades y para el aumento en los rendimientos de las variedades. El objetivo del presente fue evaluar el rendimiento de dos variedades de frijol ejotero injertadas sobre dos variedades de frijol ayocote (Phaseolus coccineous) como portainjerto. El injerto fue de tipo empalme y su prendimiento se determinó a los 20 días. A los 7, 21 y 56 días después del trasplante se midió altura de planta. En cosecha se cuantificó número de vainas, longitud de vaina y rendimiento por planta. Los resultados del porcentaje de prendimiento mostraron una temprana compatibilidad en la mayoría de los tratamientos a excepción del tratamiento FXO, que no presentó respuesta ante el injertado. Los tratamientos injertados aumentaron en $67,9 \%$ la longitud de las plantas en comparación con las plantas no injertadas, el tamaño de fruto y el rendimiento también se incrementó en 50 y 100\%, respectivamente. El frijol ejotero injertado en frijol ayocote representa una alternativa para mejorar el rendimiento y calidad del fruto.
\end{abstract}

Palabras clave: Phaseolus coccineus, injerto en hortalizas, rendimiento.

\begin{abstract}
The green beans are part of the basic diet in Latin America; however, his yield is slowed down by several factors and the growers demands new alternatives for diseases management and increase yields of varieties. The aim of this work was to evaluate the yield of two green beans cultivars grafted onto two varieties of runner bean (Phaseolus coccineous) rootstocks. The grafting was spliced side type and the success of the procedure was observed at $20^{\text {th }}$ day. At 7, 21 and 56 days after transplantation plant height was measured. At harvest, pod number, pod length and yield per plant was measured. The percentage of surviving results showed an early compatibility in most treatments except treatment FXO, provided that no response to the graft. Grafted treatments increased $67.9 \%$ in length of the plants compared with non-grafted plants, fruit size and yield also increased by 50\% and $100 \%$ respectively. The green bean grafted onto runner bean represents an alternative to improve the yield and quality of fruit.
\end{abstract}

Key words: Phaseolus coccineus, grafting vegetables, yields.

\section{Introducción}

El ejote (Phaseolus vulgaris L.) es una leguminosa que forma parte de la dieta básica de muchos países latinoamericanos; en México su producción sobrepasa el millón de toneladas por año (FAOSTAT, 2015). Sin embargo, el rendimiento del cultivo es mermado por enfermedades que lo afectan a lo largo de su ciclo productivo, entre las que destacan enfermedades como: Macrophomina sp,
Fusarium sp; Sclerotium y Rhizoctonia (ArayaFernández y Hernández-Fonseca, 2006). El control de estas enfermedades puede ser muy variado y abarca desde el aspecto cultural (manejo de podas, eliminación de residuos agrícolas, utilización de semilla certificada) hasta la utilización de productos químicos, sin embargo, los mercados actuales demandan una ecocertificación de los productos que se consumen (Orsato, 2009); esto ha llevado a los productores de hortalizas a adoptar nuevas

1 Instituto de Ciencias Agrícolas, UABC. Carretera a Delta s/n Ejido Nuevo León Mexicali B.C. México.

2 Universidad de Sonora, Departamento de Agricultura y Ganadería. Carretera a Bahía de Kino, km. 21. Hermosillo, Sonora. México.

* Autor por correspondencia: onecimo.grimaldo@uabc.edu.mx 
técnicas para el control de enfermedades, una de estas técnicas es la utilización de injertos. Entre los beneficios del injerto se encuentra una mejor toma de nutrientes por parte del sistema radicular de la planta, mejor respuesta de las plantas a déficit de agua, mejora ante altas concentraciones de salinidad en suelos, entre otros (Lee et al., 2010). Sin embargo, los objetivos principales de la técnica son la utilización de portainjertos con resistencia a las enfermedades fungosas de origen radicular y mejorar el rendimiento de la planta. En reportes de la década de 1940 se encontró que el portainjerto Cucurbita moschata (Duchesne ex. Poir) confería resistencia a enfermedades al melón (Imazu, 1949). En años más recientes se ha comprobado la eficacia de la técnica tanto en el control de enfermedades como en la mejora del rendimiento en numerosos estudios (Oda, 1995; Xu, Ma y Chen, 2014; Temperini et al., 2013; Moncada et al., 2013).

La resistencia ante enfermedades y el vigor de la planta injertada es intermedio entre el patrón y la variedad, aunque la influencia del primero es mayor que el de la variedad (Muller y Li, 2002; citado por López-Elías et al., 2008). Desafortunadamente la mayoría de los estudios en la actualidad se han centrado en la utilización de la técnica en cucurbitáceas como la sandía, melón y algunas solanáceas como el tomate y la berenjena y son pocos los estudios que se centran en otras hortalizas como el ejote.

El objetivo del presente trabajo fue evaluar el porcentaje de prendimiento del injerto y rendimiento en dos variedades del frijol ejotero injertadas sobre dos variedades de frijol ayocote como portainjerto.

\section{Materiales y Métodos}

El presente trabajo se realizó en el período de agosto a noviembre de 2015 en un invernadero tipo asimétrico de cubierta plástica, en el Instituto de Ciencias Agrícolas de la Universidad Autónoma de Baja California, localizado en el Valle de Mexicali $32^{\circ} 24^{\prime} 34$ Norte y $115^{\circ} 11^{\prime} 16$ Oeste.

Las variedades frijol ejotero utilizadas fueron Mantra y Fasili y en el caso de frijol ayocote (Phaseolus coccineus) las variedades 'Otumba' y 'CP' como portainjertos . La combinación de los materiales de frijol ejotero y ayocote generó las combinaciones descritas en la Tabla 1 donde, además, se incluye plantas sin injerto en las variedades de ejotero. El diseño experimental utilizado fue completamente al azar con 6 tratamientos y 5 repeticiones, cada planta injertada se consideró como una repetición.

La obtención de planta injertada se inició con la siembra el 10 de agosto del 2015, en charolas de 128 cavidades. Los injertos se realizaron a los 19 y 20 días después de la siembra. El procedimiento para realizar el injerto fue efectuar un corte 6 centímetros a partir de la base del tallo en un sentido de 45 grados en el portainjerto y, posteriormente, en la variedad se realizó el corte pero en sentido inverso, y esta vez por debajo del ápice de crecimiento se unieron ambos cortes con pinzas plásticas para injerto según la técnica descrita por Lee et al., 2010.

Las plantas injertadas se mantuvieron en una cámara de aclimatación con una humedad relativa (HR) de $96 \%$ y posteriormente se disminuyó gradualmente hasta llegar a una HR de $50 \%$, cinco días después se mantuvieron en condiciones de invernadero para su posterior trasplante.

El establecimiento del experimento en campo se inició a los 21 días después del injertado del ejote y consistió en trasplantar las plantas en bolsas plásticas para maceta, las cuales fueron llenadas con $1.300 \mathrm{~kg}$ de mezcla de sustrato (berger BM2 en un $60 \%$ y un $40 \%$ de arena), colocando una planta por maceta y 5 macetas por tratamiento, las macetas se colocaron a $15 \mathrm{~cm}$ de separación y las plantas fueron tutoradas con rafia. La fertilización fue en base a la fórmula convencional 120-60-160 mediante riego por goteo.

Las variables evaluadas fueron porcentaje de prendimiento del injerto a los 17 días de realizado el injerto, posteriormente a los 7, 21 y 56 días de realizado el trasplante se midió altura de planta. En cosecha se cuantificó, numero de vainas, longitud de vainas y rendimiento total por planta.

Tabla 1. Tratamientos evaluados de plantas injertadas y no injertadas.

\begin{tabular}{lc}
\hline Tratamiento & Clave \\
\hline MANTRA & $\mathrm{M}$ \\
MANTRA X OTUMBA & MXO \\
MANTRA X CP & MXCP \\
FASILI & F \\
FASILI X CP & FXCP \\
FASILI X OTUMBA & FXO \\
\hline
\end{tabular}


Los análisis de los resultados se realizó con análisis de varianza y prueba de medias para discriminar tratamientos mediante la prueba de Tukey $(0,05 \%)$. Se utilizó el software estadístico SAS 9.3 ® y Microsoft Excel 2013.

\section{Resultados y Discusión}

Los porcentajes de prendimiento de los injertos variaron entre portainjertos y variedades de ejote empleadas (Tabla 2). Los mayores porcentajes de prendimiento fue para la combinación FXCP (95\%), mientras que en la combinación FXO $(0 \%)$ no se registró prendimiento. Vrsic et al. (2015) mencionan que la compatibilidad temprana o en etapas de desarrollo del cultivo depende de factores tanto ambientales, de manejo del injerto o incluso de la combinación portainjerto-variedad. En este caso, debido a que las condiciones fueron similares para todos los tratamientos se podría indicar la presencia de incompatibilidad en la interacción entre las variedades.

En la variable altura de planta se observó que las plantas mantuvieron una altura similar hasta los 28 días de trasplante y a partir de este día el comportamiento de crecimiento fue mayor para la combinación FXCP. A los 56 días los tratamientos

Tabla 2. Porcentaje de sobrevivencia de plantas injertadas de Phaseolus vulgaris L. sobre Phaseolus Coccineous.

\begin{tabular}{lc}
\hline Tratamiento & Porcentaje de prendimiento $(\%)$ \\
\hline M & 100 \\
MXO & 70 \\
MXCP & 45 \\
F & 100 \\
FXO & 0 \\
FXCP & 95
\end{tabular}

El porcentaje de sobrevivencia fue calculado 17 días después del proceso de injertado, para esto se desprendió la pinza plástica.

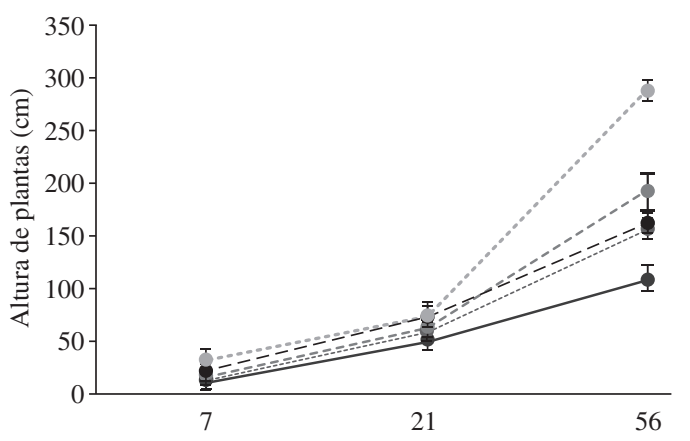

Desarrollo de plantas injertadas y no injertadas en días

$$
\begin{aligned}
& \longrightarrow-\mathrm{M} \quad \cdots \bullet \mathrm{MXO} \quad-\bullet-\cdot \mathrm{F} \\
& -\bullet \cdot \mathrm{MXCP} \quad \cdots \bullet \cdot \mathrm{FXCP}
\end{aligned}
$$

Figura 1. Altura de plantas injertadas y no injertadas de Phaseolus vulgaris L.

de planta injertada incrementaron, en promedio el $67,9 \%$ la altura. El tratamiento sobresaliente fue FXCP el cual registró $80 \%$ más de altura con respecto al testigo mientras que el tratamiento MXCP solo un 47,7\% (Figura 1). Los resultados concuerdan con los de otros investigadores en otro tipo de hortalizas (López-Elias et al., 2008; Liu et al., 2009) en los cuales se reportan un mayor desarrollo en plantas de sandía y plantas de tomate respectivamente.

Para el número de vainas promedio por planta no se encontraron diferencias entre los tratamientos; sin embargo, para el largo de las vainas se incrementó en más de $10 \mathrm{~cm}$ para el injerto FXCP en comparación con su variedad sin injertar. La superioridad del tratamiento FXCP también se vio reflejada en el rendimiento total por planta, donde se obtuvo mayor efecto en el rendimiento con respecto a los demás tratamientos (Tabla 3). Indicando una mejora en el rendimiento en un $100 \%$ y en tamaño de fruto de $50 \%$, en comparación con su respectivo testigo e incluso en comparación con otras combinaciones, resultados

Tabla 3. Variables evaluadas en los tratamientos de Phaseolus vulgaris L injertado y sin injertar.

\begin{tabular}{lccc}
\hline Tratamiento & Numero de vainas promedio por planta (por corte) & Largo de vainas $(\mathrm{cm})$ & Rendimiento por planta (g) \\
\hline FXCP & $4 \mathrm{a}$ & $33,6 \mathrm{a}$ & $151,1 \mathrm{a}$ \\
MXO & $2 \mathrm{a}$ & $14,4 \mathrm{ab}$ & $41,4 \mathrm{~b}$ \\
MXCP & $1 \mathrm{a}$ & $5,2 \mathrm{~b}$ & $15,42 \mathrm{~b}$ \\
F & $3 \mathrm{a}$ & $20,4 \mathrm{ab}$ & $74,5 \mathrm{ab}$ \\
M & $1 \mathrm{a}$ & $6,4 \mathrm{~b}$ & $17,0 \mathrm{~b}$ \\
\hline
\end{tabular}

Los tratamientos se encuentran descritos en la Tabla 1. Los valores con literales diferentes entre líneas presentan diferencias significativas en una prueba de Tukey $\alpha=0,05$. 
similares se han encontrado en otras hortalizas como tomate, berenjena y pepino (Mahmoud, 2014; Chung y Lee, 2007; Lee y Oda, 2003).

\section{Conclusión}

El injerto de frijol ejotero en frijol ayocote, representa una oportunidad para mejorar la producción y calidad de la producción. Sin embargo, es necesario realizar más trabajos en diferentes condiciones (ambientales, tipos de suelo, extensión, enfrentamiento a enfermedades) con diferentes posibles portainjertos para su futura aplicación en la producción comercial.

\section{Agradecimientos}

A Universidad Autónoma de Baja California, por el apoyo otorgado a través de la $17^{\mathrm{a}}$ Convocatoria Interna de Proyectos de Investigación.

\section{Literatura Citada}

Araya-Fernández, C.; Hernández-Fonseca, J.C.

2006. Guía para la identificación de las enfermedades del frijol más común en Costa Rica. San José, C.R.: MAG. 44 p.

Chung, H.; Lee, J.

2007. Rootstocks for grafting in Horticulture in Korea. Korean Society for horticultural Science, 28: 162-167.

Imazu, $\mathrm{T}$.

1949. On the symbiotic affinity caused by grafting among

Cucurbitaceous species. J. Jpn. Soc. Hort. Sci., 18: 6-42.

Lee, J.; Kubota, C.; Tsao, S.; Bie, Z.; Hoyos-Echeverria, P.;

Morra, L.; Oda, M.

2010. Current status of vegetable grafting: Diffusion, Grafting technioques, automation. Scientia Horticulturae, 127: 93-105.

Lee, J.; Oda, M.

2003. Grafting herbaceous vegetable and ornamental crops. Hortic. Rev., 28: 61-124.

Liu, N.; Zhou, B.; Zhao, X.; Lu, B.; Li, Y.; Hao, J.

2009. Grafting eggplant onto tomato rootstock to suppress Verticillium dahliae infection: the effect of root exudates. HortScience, 44: 2058-2062.

López-Elias, J.; Romo, A.; Domínguez, J.

2008. Evaluación de métodos de injerto en sandía (Citrullus lanatus (Thunb.) Matsum \& Nakai) sobre diferentes patrones de Calabaza. Idesia (Chile), 26 (2): 13-18.

Mahmoud, A.

2014. Grafting as a tool to improve TYLCV-Tolerance in tomato. Journal of Horticultural Science \& Ornamental Plants, 6 (3): 109-115.
Moncada, A.; Miceli, A.; Vetrano, F.; Mineo, V.; Planeta, D.;

D' Anna, F.

2013. Effect of grafting on yield and quiality of eggplant (Solanum melongena L.). Scientia Horticulturae. 149: 108-114.

Muller, D.; Li, S.

2002. Use of aeroponic chambers and grafting to study partial resistance to Fusarium solani f. sp. Glycines in soybean. Plant Disease 86: 1223-1226.

Oda, $\mathrm{M}$.

1995. New grafting methods for fruitbearing vegetables in Japan. Japan Agricultural Res. Qrty. 29: 187-194.

Organización de las Naciones Unidas para la Alimentación y la Agricultura (Sistema Estadístico) Disponible en:http:// faostat3.fao.org/browse/Q/QC/E Consultado: 12/Dic/15

Orsato, R.

2009. Sustainable Strategies: When Does It Pay To Be Green? Palgrave Macmillan, Basingstoke, Hampshire, UK. 243 p.

Temperini, O.; Calabrese, N.; Temperini, A.; Rouphael, Y.; Tesi, R.; Lenzi, A.; Carito, A.; Colla, G.

2013. Grafting artichoke onto cardoon roostocks: Graft compatibility, yield and verticillium wilt incidence. Scientia Horticulturae, 149: 22-27.

Vrsic, S.; Pulko, B.; Kocsis, L.

2015. Factor influencing grafting success and compatibility of grape. Scientia Horticulturae, 181: 168-173.

$\mathrm{Xu}, \mathrm{C}$.; Ma, Y.; Chen, H.

2014. Technique of grafting with wufanshu (IVaccinium bracteatum Thunb) and the effects on blueberry plant growth and development, fruit yiel and quality. Scientia Horticulturae, 176: 290-296. 